\title{
GPPS-BJ-2019-0232
}

\section{INFLUENCE OF MERIDIAN FLOW PATH AND LOADING DISTRIBUTION ON THE PERFORMANCE OF TURBINE REAR FRAME}

\author{
Shulei Li \\ Collaborative Innovation Center for Advanced \\ Aero-Engine, School of Energy and Power \\ Engineering, Beihang University \\ Isl_1304@buaa.edu.cn \\ Beijing, China
}

\author{
Xinmin Gui \\ Collaborative Innovation Center for Advanced \\ Aero-Engine, School of Energy and Power \\ Engineering, Beihang University \\ guixm@buaa.edu.cn \\ Beijing, China
}

\author{
Donghai Jin \\ Collaborative Innovation Center for Advanced \\ Aero-Engine, School of Energy and Power \\ Engineering, Beihang University \\ jdh@buaa.edu.cn \\ Beijing, China
}

\author{
Jinwei Li \\ Aero Engine Academy of China \\ 13810871565@126.com \\ Beijing, China
}

\begin{abstract}
The prototype gas turbine has low efficiency and severely deviated outlet flow, thus, the Turbine Rear Frame (TRF) meridian flow path and Outlet Guide Vane (OGV) are redesigned, and the influence of meridian flow path and the different loading distribution on the internal flow field structure is analyzed by CFD calculations. The results show that when the maximum flow area ratio of the blade area is too high, separation will occur. The flow path design with a character that expand first and then contract can suppress the corner separation. The load distribution of OGV will affect the deflection distribution. Moving the maximum deflection position backwards can make the blade suction side turn smoothly, which is beneficial to reduce the axial adverse pressure gradient and relieve the suction surface corner separation. The transition-loaded airfoil can form an endwall bend on the suction side of the blade, reduce the cross flow in the channel, and alleviate the corner separation. After the retrofit design, the turbine efficiency can be increased by $6 \%$ or more, and the pressure recovery coefficient of OGV has a rise of more than 0.08 , the outlet flow deviation after OGV is less than $3.5^{\circ}\left(15.864^{\circ}\right.$ for prototype).
\end{abstract}

\section{INTRODUCTION}

Modern aero-engine design pursues high-performance design goals. The turbine has to increase the load greatly in a small number of stages. The inlet flow angle of the OGV is also increased, raising the design difficulty. Therefore, the turbine rotor outlet flow angle is likely to be severely off-axis. If the flow direction is not corrected, the engine thrust and the isentropic efficiency will be severely affected as the flow field deteriorate. The TRF is part of the engine's load-bearing system, which is often equipped with support plate as well as the OGV, who has the function of turning the outlet flow to axial with the smallest total pressure loss ${ }^{[1]}$.

Many scholars at home and abroad have carried out studies on TRF/OGV. For the numerical setup, Hjärne et al. used a combination of numerical and experimental methods to compare the turbulence model used in the numerical calculation of the TRF/OGV, analyzed the flow field and the flow field vortex structure of the TRF/OGV outlet. The performance of the non-design point of the turbine afterrectifier cascade was also studied in detail. It was concluded that the initial design suggested using a $\mathrm{k}-\varepsilon$ model with wall correction as the turbulent model, which was more accurate in the prediction of wake and loss. For the secondary flow in the TRF/OGV, Hjärne depicted the flow field in detail, and found the inlet flow angle, turbulence intensity and boundary layer thickness of the OGV had an effect on the scale and position of the secondary flow in the internal flow field ${ }^{[2,3,4]}$. To control the secondary flow in the TRF/OGV, Sonada et al. studied the OGV of the extreme front-loaded airfoil, found that under the low Reynolds number condition, it could obtain better performance than the ordinary Control Diffusion Airfoil $(\mathrm{CDA})^{[5]}$. From the pitch and the elemental airfoil aspect, Koch et al. optimized an OGV and conducted numerical and 
experimental studies on an OGV of a low-pressure turbine, and analyzed the flow field performance at different inlet angles under low Reynolds number conditions ${ }^{[6]}$. Yan took the installation joint structure into consideration and conducted a numerical simulation of the low-pressure turbine OGV, and analyzed the influence of design parameters and the installation joint structure, then completed the aerodynamic design of a low-pressure turbine $\mathrm{OGV}^{[7]}$. For engineering application, Zhang completed aerodynamic design of the lowpressure turbine OGV according to the given low-pressure turbine outlet conditions, and concluded the aerodynamic design method, the verified by numerical simulation ${ }^{[8]}$. Feng carried out an experimental study on the low-pressure turbine $\mathrm{OGV}$, and analyzed the influence of the inlet Mach number and the flow angle on the aerodynamic performance of the TRF/OGV. The design point and the non-design point results were compared ${ }^{[9]}$. To reduce weight as well as get best aerodynamic performance, Feng optimized the TRF/OGV using the splitter blade design ${ }^{[10]}$.

For weight reduction consideration, the integrated design OGV and the support plate is studied in this paper. The structure of the TRF prototype meridian flow path is shown in Fig.1. The OGV is not in the straight part of the flow path after the turbine rotor, but is integrated with the inner cone flow path of the nozzle, so the hub of the flow path is inclined inward. The pipeline will be installed inside the OGV, thus the airfoil of the OGV has to have a long blade chord length, a small aspect ratio.

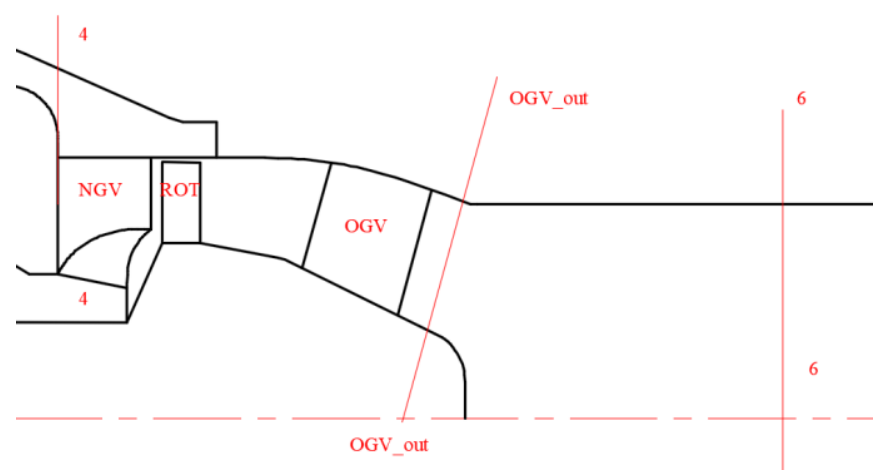

\section{Figure 1 Flow Path Structure and Section Definition of Turbine}

Remaining the axial length of the TRF unchanged, this paper uses the numerical simulation method to modify the design of TRF/OGV to improve the turbine efficiency and obtain a axial outlet flow, modifying flow path and the blading loading profiles to control the secondary flow in the flow field, which provides a reference for the integrated design of similar TRF/OGV.

\section{RESEARCH METHOD AND NUMERICAL SETUP}

In this paper, the commercial software NUMECA is used to numerically simulate the single-stage turbine and the TRF/OGV. The generation of OGV is carried out by using autonomously developed software for blade shaping by a given thickness distribution, number of blades, flow angle distribution, maximum thickness position and deviation angle distribution. The number of blades of the Nozzle Guide Vane (NGV) is 25, the number of blades of Rotor (ROT) is 52. The grid is generated by Auto Grid 5, and as the total number of grids reaches 1.13 million, the number of grids in OGV reaches 0.45 million, the aerodynamics performance hardly changes, the grid that satisfied the grid independency is shown in Fig. 2. The calculation fluid type follows the gas in the reference [11]. Using RANS method with the S-A turbulence model. The 1 st level $y+$ has a value lower than 10 and use the conservative coupling by pitchwise row approach as the rotorstator interface. The design point inlet total temperature of $1197.51 \mathrm{~K}$, total pressure of $521400 \mathrm{~Pa}$, and the flow is given axial intake, while the ROT speed is given 22000rpm. The outlet given the average static pressure, the initial calculation is given uniform axial flow of $140 \mathrm{~m} / \mathrm{s}$.

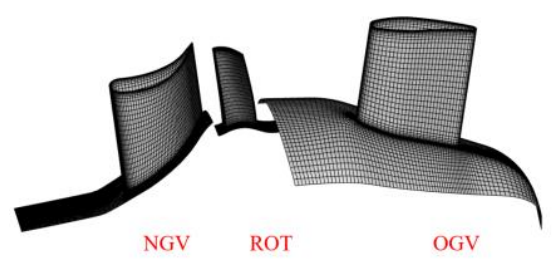

Figure 2 The Mesh Structure

Table 1 Grid Independency Validation

\begin{tabular}{|c|c|c|}
\hline $\begin{array}{c}\text { Total grid numbers } \\
\text { /million }\end{array}$ & $\begin{array}{c}\text { Total pressure recovery } \\
\text { coefficient in OGV }\end{array}$ & $\begin{array}{c}\text { Isentropic efficiency of } \\
\text { turbine (include OGV) }\end{array}$ \\
\hline 0.84 & 0.8955 & 0.8122 \\
\hline 0.99 & 0.8944 & 0.8111 \\
\hline 1.13 & 0.8934 & 0.8102 \\
\hline 1.20 & 0.8927 & 0.8105 \\
\hline
\end{tabular}

\section{NUMERICAL VALIDATION}

In the experiment, as $\mathrm{p}_{6}$ and $\mathrm{p}_{8}$ is determined by the environment, a low total pressure recovery coefficient $\left(\sigma_{\mathrm{OGV}}\right)$ in OGV result in the rise in $\mathrm{p}_{\mathrm{t} 5}$, the power of turbine can't change as the rotating speed does not change, consequently, the fuel requirement in the combustor rise, making the inlet total pressure and temperature change. Yet, in the numerical simulation the inlet condition is set unchanged, thus the low total pressure recovery coefficient in OGV result in a lower $\mathrm{p}_{\text {t6. }}$. Thus, the inlet conditions are changed to match the experiment point, the parameter and the relative error are shown in Table 2.

\section{Table 2 The Comparison of Experiment and} Numerical Results

\begin{tabular}{|c|c|c|c|}
\hline ISA22000rpm & Experiment & Simulation & Relative Error \\
\hline Mass Flow $/(\mathrm{kg} / \mathrm{s})$ & 14.15 & 14.08 & $0.49 \%$ \\
\hline $50 \%$ Span $\mathrm{T}_{\mathrm{t} 6} / \mathrm{K}$ & 1050.40 & 1051.28 & $0.08 \%$ \\
\hline $48 \%$ Span $\mathrm{p}_{\mathrm{t} 6} / \mathrm{kPa}$ & 177.787 & 190.399 & $7.09 \%$ \\
\hline $64 \%$ Span $\mathrm{p}_{\mathrm{t} 6} / \mathrm{kPa}$ & 189.913 & 194.977 & $2.67 \%$ \\
\hline $50 \%$ Span $\sigma_{\mathrm{OGV}}$ & 0.872 & 0.876 & $0.05 \%$ \\
\hline
\end{tabular}

According to Table 2, the maximum relative error is $7.09 \%$, which validates the simulation result. The comparison of spanwise distribution of the turbine rotor outflow angle is shown in Fig. 3. The flow angle is defined by the angle 
between the axial direction and the direction of the flow, which is the same as the positive direction of the rotor, and vice versa.

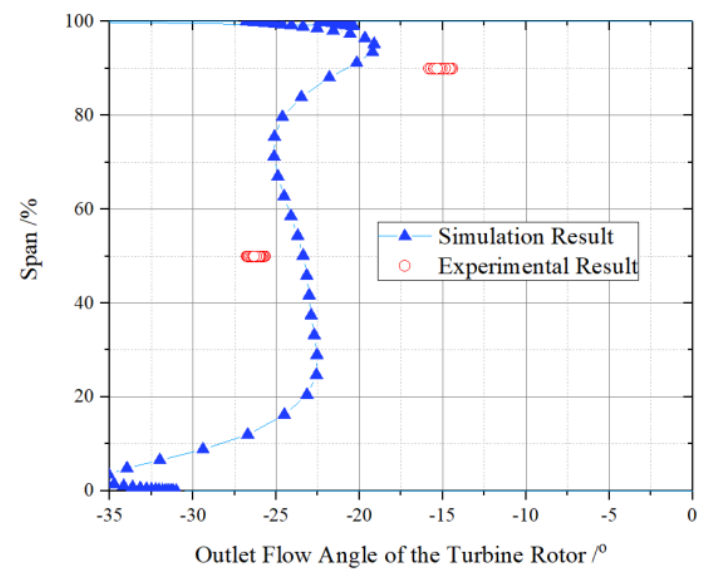

Figure 3 the Spanwise Distribution of Turbine Rotor Outflow Angle

According to Fig.3, the outlet flow angle in $50 \%$ span has an error of proximately 3 degree, and in $90 \%$ span the error is 5 degree. The possible reason for the error in the experiment and the numerical simulation result is that the simulation result is a circumferential average result, yet the experimental measure position is at a particular circumferential position. Due to the influence of vane, the outflow angle after rotor may be slightly different, let alone the real flow field in the experiment is unsteady, the wake may also have an effect on the result.

In a cascade channel, there is a cross flow in both the endwall due to the pressure gradient from the pressure surface to the suction surface. Therefore, the flow at the hub deviates more in the negative direction than the intermediate flow. The ROT with a $0.52 \mathrm{~mm}$ gap calculation has a tip leakage flow with a positive flow direction, so the tip flow deviates more in the positive direction than the intermediate flow. It can be seen that the numerical simulation results are consistent with the actual flow mechanism, which is a credible result. The inlet flow angle design of OGV can be given according to the numerically calculated extension distribution law at the design point.

\section{RESULTS AND ANALYSIS}

\section{The Aerodynamic Performance of TRF/OGV of the Prototype}

The results of numerical simulation of the prototype turbine at the design point shows that there are large outlet flow loss and swirling at the exit, which has a significant influence on efficiency. The prototype turbine aerodynamic performance parameter is given in the Table 3 .
Table 3 Results of the Prototype at Design Point

\begin{tabular}{|c|c|}
\hline ISA 22000rpm & Prototype simulation \\
\hline $\mathrm{T}_{\mathrm{t} 4} / \mathrm{K}$ & 1197.51 \\
\hline $\mathrm{p}_{\mathrm{t} 4} / \mathrm{kPa}$ & 521.4 \\
\hline Mass flow $/ \mathrm{kg} / \mathrm{s}$ & 13.46 \\
\hline Gas turbine stage efficiency & 0.837 \\
\hline Isentropic turbine efficiency (include OGV) & 0.810 \\
\hline$\sigma_{\mathrm{oGV}}$ & 0.893 \\
\hline $\mathrm{T}_{\mathrm{t} 6} / \mathrm{K}$ & 978.22 \\
\hline $\mathrm{p}_{\mathrm{t} 6} / \mathrm{kPa}$ & 178.9 \\
\hline Flow angle of section $6 /{ }^{\circ}$ & -15.864 \\
\hline
\end{tabular}

Fig.4 shows the spanwise distribution of turbine rotor outflow angle at the design point and the design input flow angle of OGV. In order to prevent the corner separation on the suction side of OGV, the design input of OGV derivate $-3^{\circ}$ from the simulation result of the prototype at the design point at $50 \%$ span.

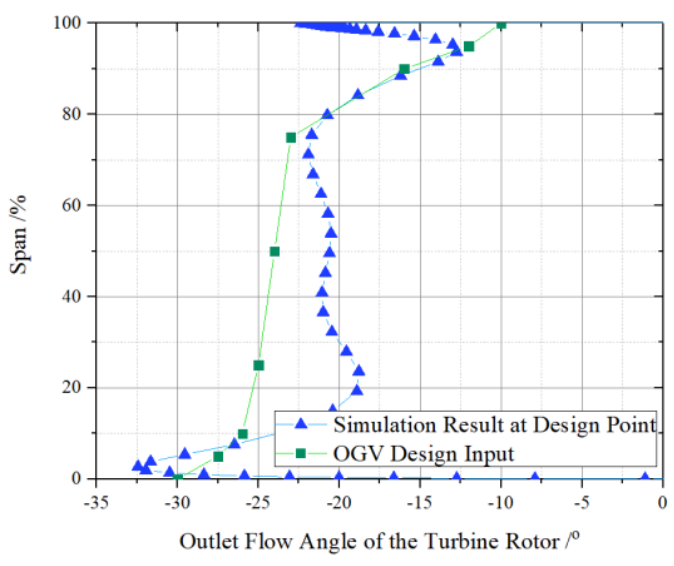

Figure 4 the Spanwise Distribution of Turbine Rotor Outflow Angle

\section{The Influence of Meridional Flow Path on Aerodynamic Performance}

In this paper, four type of flow path is designed, and 12 stations are set along the flow direction. Fig. 4 shows the structure of different meridional flow path designs. Each station is represented by a purple line, and the red calculation station position indicates the blade leading edge and trailing edge. Since the hub is an inner cone structure, the area of meridional flow path inevitably expands. The expansion of the channel flow area after the maximum thickness of the airfoil interacts with the flow path expansion, causing adverse pressure gradient to increase, thus separation is easily to be induced.

The airfoil of the TRF/OGV is CDA077, the maximum thickness is $6.4 \%$ chord length, the maximum thickness position is $30 \%$ axial position, and the number of blades is set to 10. The flow area ratio is defined as the Area/ Area_inlet in the OGV.

The shroud in design A, B and C are the same, while the shroud in design $\mathrm{D}$ changes to make the flow area expanding continuously. 


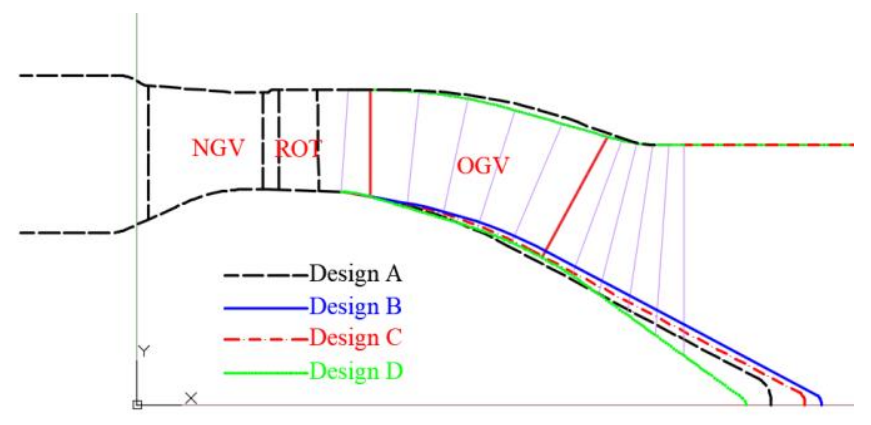

Figure 5 The Meridian Flow Path of the Blade Area

\section{Table 4 Parameter Comparison of Different Meridional Flow Path Design}

\begin{tabular}{|c|c|c|c|c|c|}
\hline ISA 22000rpm & Prototype & $\mathrm{A}$ & $\mathrm{B}$ & $\mathrm{C}$ & $\mathrm{D}$ \\
\hline $\begin{array}{c}\text { Maximum flow area ratio } \\
\text { in the OGV blade region }\end{array}$ & & 1.088 & 1.053 & 1.024 & 1.022 \\
\hline $\begin{array}{c}\text { Isentropic turbine } \\
\text { efficiency (include OGV) }\end{array}$ & 0.810 & 0.862 & 0.864 & 0.875 & 0.874 \\
\hline$\sigma_{\mathrm{ogV}}$ & 0.893 & 0.961 & 0.963 & 0.977 & 0.975 \\
\hline $\mathrm{T}_{\mathrm{t} 6} / \mathrm{K}$ & 978.22 & 978.23 & 978.24 & 978.21 & 978.24 \\
\hline $\mathrm{p}_{\mathrm{t} 6} / \mathrm{kPa}$ & 178.9 & 192.5 & 192.9 & 195.8 & 195.4 \\
\hline Flow angle of section $6 /^{\circ}$ & -15.864 & -7.203 & -6.924 & -6.134 & -6.752 \\
\hline
\end{tabular}

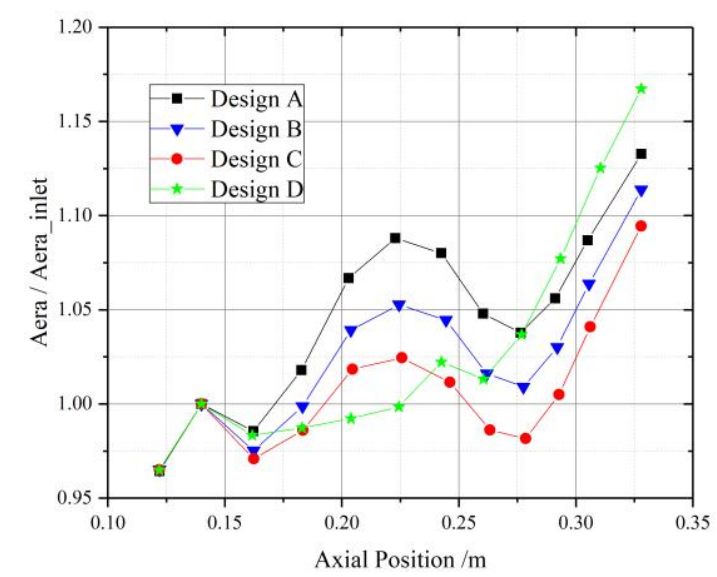

\section{Figure 6 The Distribution of Blade Flow Area}

The maximum value of the flow area ratio of the blades in the blade area from A to $\mathrm{C}$ is successively decreased. The flow area ratio of these three designs increases first and then decreases along the flow direction, and then increases again after leaving the blade area. The blade flow area ratio of design $\mathrm{C}$ is 0.02 higher than design $\mathrm{D}$ (1.022), but the $\mathrm{D}$ design keeps the area growing throughout the blade area, reaching the maximum in the blade trailing edge station. Due to the smooth and continuous requirements of the flow path profile, the Ddesigned blade flow area is the largest with increasing gradient, and the value reached at the last calculation station is also the largest. The maximum value of flow area ratio and aerodynamic performance of the four designs are given in Table 4.
It can also be seen from Table 4 that although the D design blade has the lowest maximum value of flow area ratio, design $\mathrm{C}$ has the highest efficiency of turbine (include OGV), which is $6.5 \%$ higher than the prototype, the total pressure recovery coefficient of the OGV is also the largest, which is 0.084 higher than the prototype.

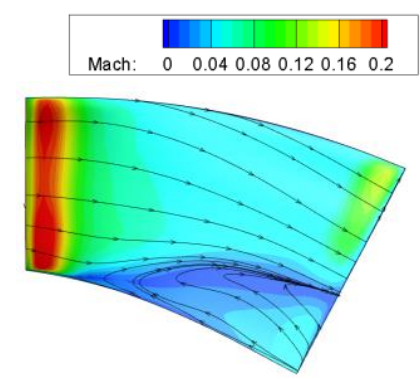

(a) Meridian Flow Path Design A

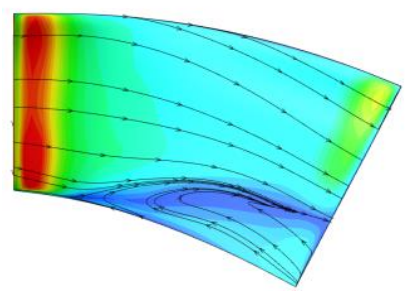

(b) Meridian Flow Path Design B

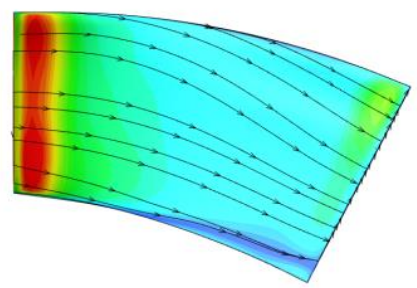

(c) Meridian Flow Path Design C

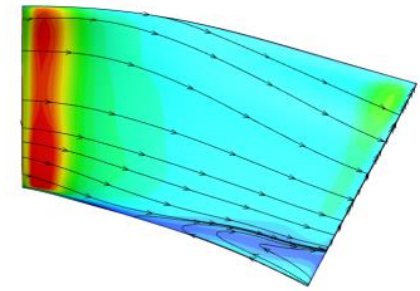

(d) Meridian Flow Path Design D

\section{Figure 7 The Suction Side of Different Airfoil under Different Meridian Flow Path Design}

Fig. 7 shows the surface streamline of the suction side of the OGV of each flow path design. It can be seen that since the flow path is a downward contraction curve, the hub is equivalent to the inner tube wall of the pipe. The flow separation is likely to occur during the turn-down process. At the same time, as the blade flow area ratio decreases, that is, the adverse pressure gradient decreases, the scale of the suction surface corner vortex is smaller. Flow path design A and $\mathrm{B}$ all have a separation near the hub of suction side. The corner separation of design A occupies an area of about $45 \%$, while design B only about $40 \%$. No separation is found in design $\mathrm{C}$ with a maximum flow area ratio of 1.024 . Compared 
with the design $\mathrm{C}$, although the maximum value of the flow area in the blade area is smaller, there is still a suction corner separation of about $15 \%$ span in Design D. The results indicate that an appropriate reduction of the flow area at the trailing edge of the blade is beneficial to suppress the separation of the corner area of the suction side. The essence is to accelerate the low velocity flow in the corner zone by using a positive pressure gradient, thereby suppressing the separation.

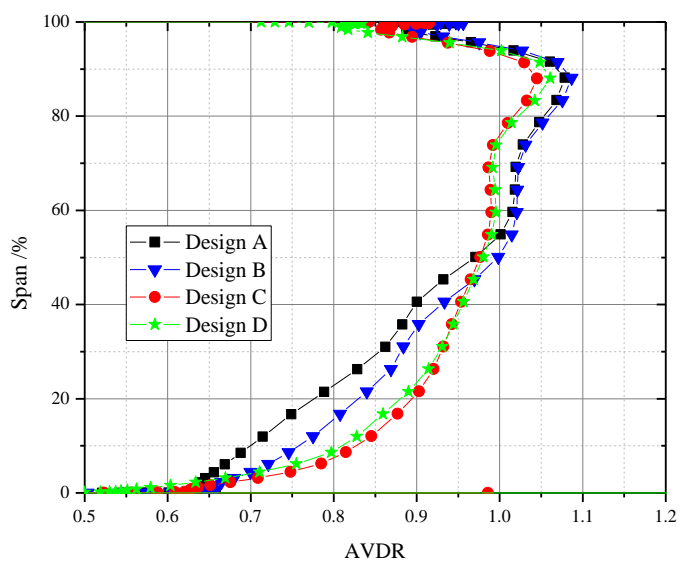

\section{Figure 8 Comparison of AVDR Results Calculated by Grid}

Fig. 8 shows the Axial Velocity Density Ratio (AVDR) of each meridional flow path design calculated according to the grid. It can be seen that with the maximum value of the blade flow area ratio decreasing, the corner separation diminishes, the value of AVDR near hub is improved. When the maximum flow area ratio of the blade is less than 1.053, the angle of the suction surface of the turbine is reduced to three-dimensional separation, the AVDR near hub increases, and the intermediate span AVDR is decreased. Due to the upward contraction of the flow path at the trailing edge of the design $\mathrm{C}$, the AVDR near hub is improved comparing to design D. According to the results published by Kumaran in $2015^{[12]}$, a large separation area would occur in the suction side of a CDA airfoil when the AVDR below certain value, which revealed a linkage between the minimum AVDR and the blockage. A low AVDR area can be seen near the hub of OGV, reflecting a blockage zone at corner zone, which is likely to induce corner separation. Yet, due to the low aspect ratio, the separation line is not easy to appear in the flow field; at the same time, if strictly calculated according to the flow tube, the intermediate span AVDR should be higher, and the AVDR in hub and shroud should be lower.

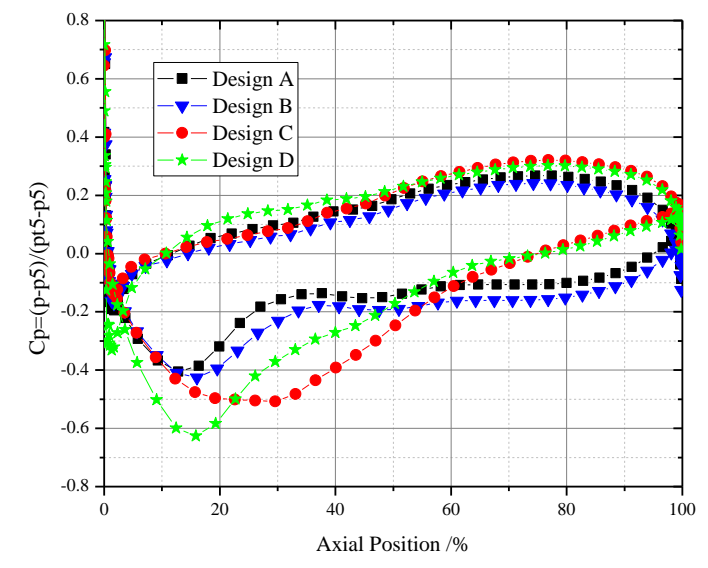

Figure 9 Static Pressure Coefficient Distribution on Blade Surface on $10 \%$ Span

Fig.9 shows the comparison of the surface static pressure coefficient distribution ( $\mathrm{Cp}$ ) of each flow path design. It can be seen that the design A has a plateau area at the axial position of $30 \%$ on the suction surface, in which the pressure does not continue to increase. This is caused by the separation of the suction surface. The position of the platform area is postponed to about $35 \%$ axial position in design B. It is verified that the reduction of the maximum flow area of the blade can make the corner separation of the suction surface smaller. The pressure of the design $\mathrm{C}$ suction surface increases more uniform than design $\mathrm{D}$, and the lowest value achieved by the static pressure coefficient is higher than the $\mathrm{D}$ design, thus design $\mathrm{C}$ is a better design. It is proved again that if the flow area of the blade increases first and then decreases, it can alleviate the adverse pressure gradient of the trailing edge of the suction surface, which is beneficial to the control of the corner vortex.

In summary, when the maximum flow area ratio of the blade section is greater than or equal to 1.053 , a corner separation appears in the suction side of OGV, which affects the total pressure recovery coefficient of the OGV. When the maximum flow area ratio of the blade area is less than or equal to 1.024 , if the flow area of the blade expands first and then contract, the three-dimensional corner separation of the suction surface can be eliminated; if the flow area of the blade expand gradually, the total pressure recovery coefficient of OGV is reduced by 0.002 , and there is a three-dimensional corner separation in the range below the $15 \%$ span of the trailing edge of the suction side. This is because a contracting flow path can be generated at the hub of the trailing edge of $\mathrm{OGV}$ in the former profile, which is beneficial to reduce the adverse pressure gradient and eliminate the corner separation. 


\section{Influence of TRF/OGV Load Distribution on Aerodynamic Performance}

As mentioned in Reference [1], after the maximum thickness position of the TRF/OGV, due to the rapid decrease of the thickness of the airfoil, the area of the second half of the flow path is rapidly expanded, and there is a strong adverse pressure gradient in the flow field. Therefore, the use of the front-loaded airfoil can lengthen the axial length of the adverse-pressure gradient, reduce the adverse pressure gradient per unit length, and facilitate the suppression of separation ${ }^{[1]}$. However, in the case of this paper, the airfoil near the hub needs to be uniform-loaded to effectively control the corner separation of the suction side. The flow in the corner of the suction side of the blade is mainly affected by the axial pressure gradient and the lateral pressure gradient between the pressure and suction side. The load distribution form of OGV will change the position of the maximum deflection, and the maximum deflection position and the maximum thickness position should be matching to form a gentle pressure rise of the suction surface. At the same time, since the OGV applies a gravity stack, applying different load distribution forms from the root to the tip may cause the blade to bend partly and affect the lateral flow between the pressure and suction side.

In this paper, by giving the same inlet and outlet flow angle, the blade load pattern is controlled by a given flow angle in the blade modeling program. The flow angle on the nine span positions are given to determine the shape of the blade. The meridional flow path uses design $\mathrm{D}$ in the former chapter, and the elementary airfoil type still uses the CDA077. Table 1 shows the maximum deflection positions of OGV in each of the three load distribution forms. The airfoil design I shown in Fig.11(a) is all front-loaded from root to tip. As shown in Fig.11(b), the airfoil design II is uniform-loaded from the root to the tip. The airfoil design III shown in Fig.11(c) is a transitional-loaded airfoil type with uniformloaded in the root and front-loaded in the tip.

Table 5 Comparison of the Maximum Deflection Positions of Different Airfoil Design

\begin{tabular}{|c|c|c|c|}
\hline \multirow{2}{*}{ Span/\% } & \multicolumn{3}{|c|}{ Relative location of the Max deflection } \\
\cline { 2 - 4 } & Airfoil design I & Airfoil design II & Airfoil design III \\
\hline 0 & 0.3820 & 0.5176 & 0.5176 \\
\hline 5 & 0.3820 & 0.5151 & 0.4996 \\
\hline 10 & 0.3818 & 0.5133 & 0.4810 \\
\hline 20 & 0.3811 & 0.5107 & 0.4606 \\
\hline 50 & 0.3788 & 0.5053 & 0.4213 \\
\hline 80 & 0.3774 & 0.5008 & 0.3774 \\
\hline 90 & 0.3775 & 0.4972 & 0.3775 \\
\hline 95 & 0.3775 & 0.4957 & 0.3775 \\
\hline 100 & 0.3774 & 0.4948 & 0.3774 \\
\hline
\end{tabular}

Fig. 11 shows the suction side for three airfoil designs. It can be seen that the acceleration of the airfoil design I is the most rapid, the leading edge acceleration and deceleration zones of the airfoil design II are the most moderate, and the characteristics of the airfoil design III are between the two. Because the front-loaded design in airfoil I, the maximum deflection position lies in the most front place, the acceleration length of the suction front edge is the shortest, so acceleration is rapid. The blade design II with the uniform-loading design accelerates moderately on the suction side leading edge. The characteristics of the airfoil design III are somewhere in between. In the airfoil design I, a separation begins to emerge from about $20 \%$ of the axial position of the root, and develops into a vortex. At the trailing edge, the impact of the separation has expanded to about $40 \%$ span. In the airfoil design II, the low-speed zone generates from about $45 \%$ of the axial position of the root, and occupies about $20 \%$ of the extension to the trailing edge. A certain backflow occurs in the $60 \%$ to $100 \%$ axial position of the root in the airfoil design III, the root begins to develop a low-speed zone from about $20 \%$ of the axial position, but only develops to about $20 \%$ of the height at the trailing edge.

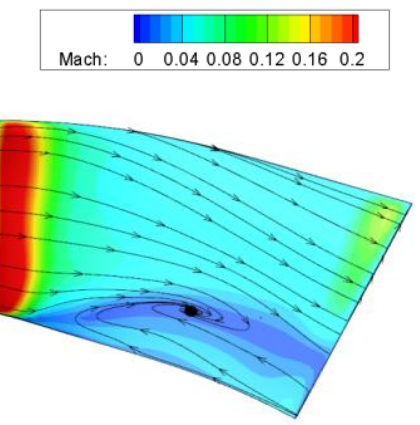

(a) Airfoil design I

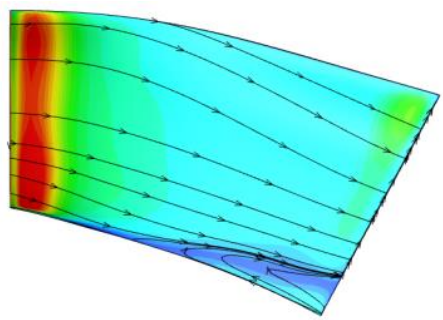

(b) Airfoil design II

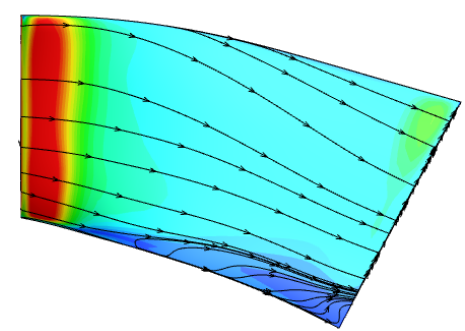

(c) Airfoil design III

\section{Figure 11 The Suction Side of Different Airfoil}

According to the control principle to the corner vortex of the enwall bending airfoil, the local positive bending at the root $^{[13]}$ can reduce the lateral pressure gradient and inhibit the radial migration of the low energy fluid in the corner, thus weakening the corner separation. The results in Fig. 11 prove this point. In the airfoil design I, due to the corner separation of the suction side, the streamline clearly leaves the blade wall and the static pressure distribution is also affected.

As for airfoil design II and III, It can be clearly seen that the lateral pressure gradient of the airfoil design III from the pressure side to the suction side is reduced, the static pressure contour is also more sparse, and the radial flow of the low 
energy fluid at the trailing edge of the suction side is suppressed, so the corner separation is weakened. At the same time, Fig. 12 also shows that the corner separation is affected by the lateral pressure gradient between the pressure side to the suction side as well as the axial adverse pressure gradient, wherein the axial pressure gradient is higher, and plays a major role in it.

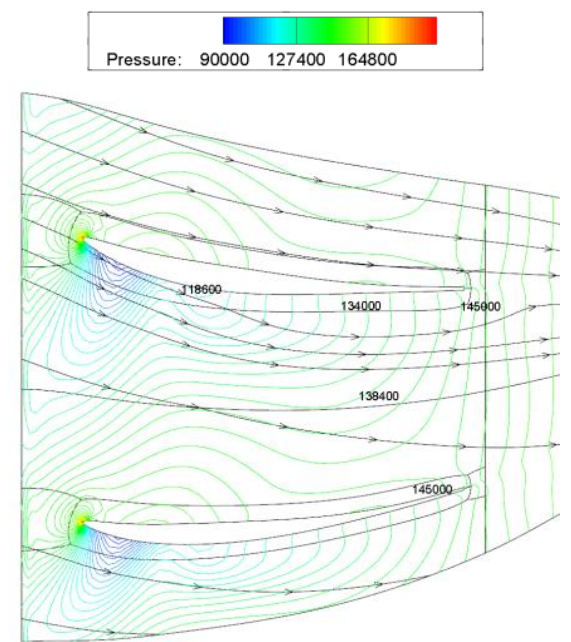

(a) Airfoil design I

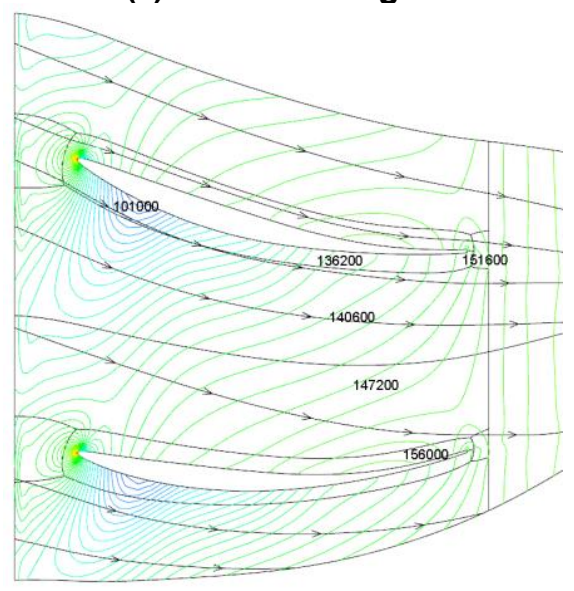

(b) Airfoil design II

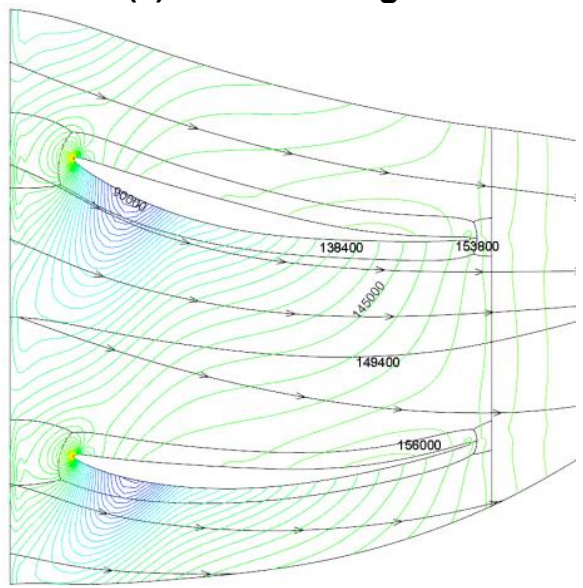

(c) Airfoil design III

Figure 12 Pressure and Streamline Distribution on $10 \%$ Span on Flow Surface S1
Fig.13 shows the distribution of the total pressure recovery coefficient of the blade area along the span in the three blade load distributions airfoil designs. It can be seen that the airfoil design I exhibits a distinct low total pressure recovery coefficient region at span $15 \%-50 \%$, corresponding to the separation of the flow shown in the former diagram. Due to the separation of the airflow, the flow tube is contracted upwards and the air flow is squeezed to a higher elevation position, so this design has the highest total pressure recovery coefficient in the 50\%-60\% span. In the region below $20 \%$ span, the total pressure recovery coefficient of the airfoil design III is the highest, which is related to the positive bending of the airfoil design at the root. In the span range of $60 \%-100 \%$, the total pressure recovery coefficients of the three airfoil designs are not much different.

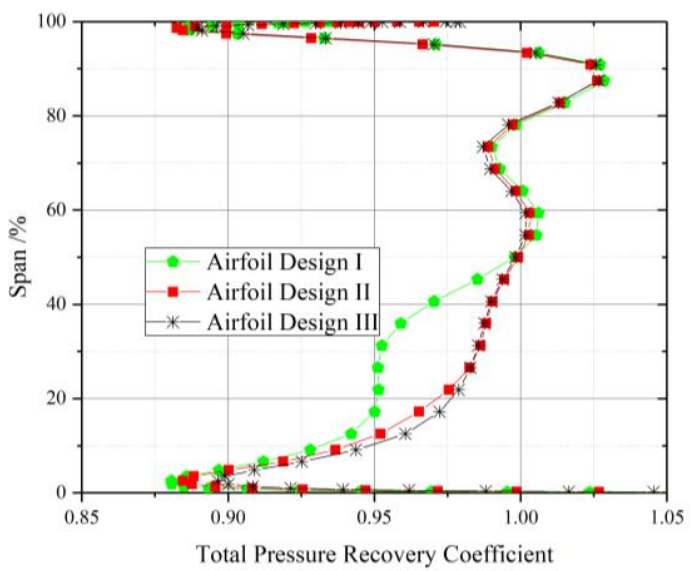

\section{Figure 13 Comparison of Total Pressure Recovery Coefficient of OGV}

Fig. 14 shows the distribution of the outlet flow angle for the three blade loading forms. It can be seen that the airfoil design II has the largest deviation angle over the entire span range. Below the $70 \%$ span, the absolute value of the outlet flow angle of the airfoil design I is smaller than that of the airfoil design III. In the airfoil design I, the flow separation in the root zone of the suction surface occupies almost $40 \%$ of the area where the flow is not controlled by the airfoil, thus the outlet flow appears closer to the axial direction.

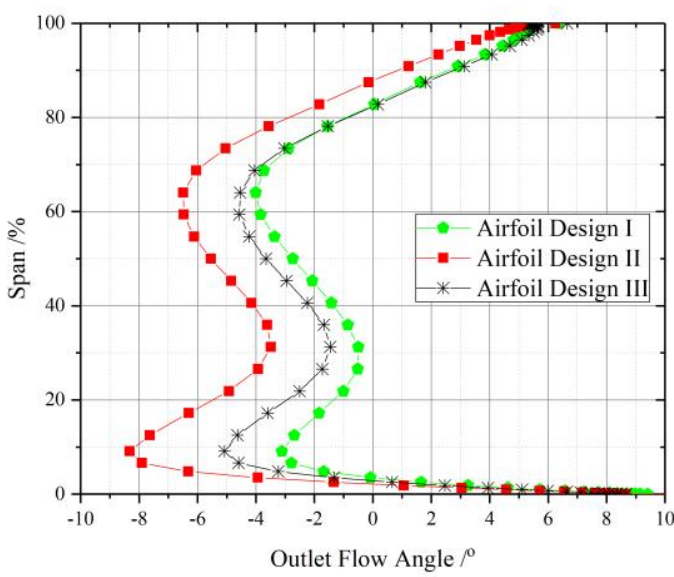

Figure 14 Comparison of Outlet Flow Angle of OGV 


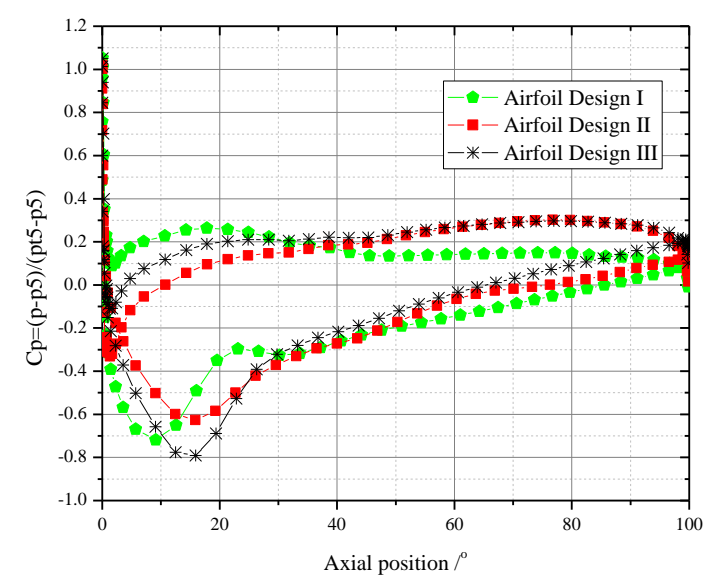

\section{Figure 15 Comparison of Pressure Coefficient of OGV on $10 \%$ Span}

Fig. 15 shows the distribution of the $10 \%$ span static pressure coefficient of the three blade load distribution patterns. It can be clearly seen that the airfoil design I has a plateau area at $20 \%$ axial position, corresponding to the start position of separation. Due to the existence of separation, the static pressure at the trailing edge does not reach the same level as the airfoil design II and III. The static pressure of the suction surface of the airfoil design II is the most uniform, but the acceleration in the leading edge of the suction side of airfoil III is the most intense, and the pressure is increased rapidly at the $20 \%-30 \%$ axial position. The subsequent pressure rise is gentle; thus, this airfoil design still needs to be improved.

Table 6 Comparison of Different Airfoil Design

\begin{tabular}{|c|c|c|c|c|}
\hline ISA 22000rpm & Prototype & Design I & Design II & Design III \\
\hline $\begin{array}{c}\text { Relative location of the } \\
\text { Max deflection /\% }\end{array}$ & & 0.3788 & 0.5053 & 0.4213 \\
\hline $\begin{array}{c}\text { Isentropic turbine } \\
\text { efficiency (include OGV) }\end{array}$ & 0.810 & 0.865 & 0.874 & 0.874 \\
\hline$\sigma_{\text {oGv }}$ & 0.893 & 0.964 & 0.975 & 0.976 \\
\hline $\mathrm{T}_{\mathrm{t} 6} / \mathrm{K}$ & 978.22 & 978.21 & 978.24 & 978.24 \\
\hline $\mathrm{p}_{\mathrm{t} 6} / \mathrm{kPa}$ & 178.9 & 193.1 & 195.4 & 195.4 \\
\hline Flow angle of section 6 / & -15.864 & -2.385 & -6.752 & -3.474 \\
\hline
\end{tabular}

Table 6 shows the relative axial position of maximum deflection on span $50 \%$ and basic performance parameters of the three airfoil designs. It can be seen that the OGV with the front-loaded blade type (airfoil design I) has a large internal separation, thus its total pressure recovery coefficient is the lowest. When the uniform-loaded type (airfoil design II) is adopted, the maximum deflection position is too far back, the control of the flow steering is reducing, and the 6-section outlet flow angle derivate the most. When the transitionloaded blade type with the tip area front-loaded and the root area uniform-loaded (airfoil design III) is adopted, the turbine efficiency (include OGV) can be improved by $6.4 \%$, the total pressure recovery coefficient of OGV reaches 0.976 , which is 0.083 higher than the prototype, the outlet flow deviation after OGV is less than $3.5^{\circ}\left(15.864^{\circ}\right.$ for prototype).
In summary, the uniform-loaded airfoil (airfoil design II) gives the lowest load on the position where maximum thickness of the blade lies, thus it is the gentlest turning airfoil. The axial gradient of OGV is the lowest, which is beneficial to eliminate the corner separation, but the ability to correct the deviation of the outlet flow angle declines. The front-loaded blade type (airfoil design I) has a separation zone at the root of the suction surface. So when applying a transition-loaded airfoil design in which the tip of the tip is front-loaded and gradually transits to uniform-loaded in the root (airfoil design III), the maximum deflection position of the blade is in the middle of the two former designs, making the suction surface shape turning more smoothly. While increasing the axial length of undertaking the adverse pressure gradient comparing to airfoil design II, it can suppress the separation of the corners on the suction side and optimally control the direction of the outlet flow. At the same time, due to the change of the blade load distribution forms, the trailing edge of the root is deflected toward the suction surface to form the end bend, which has an influence on the lateral pressure gradient of the cascade channel, and has a good inhibitory effect on the suction surface vortex.

\section{CONCLUSION}

In this paper, a redesigned performance of an integrated design of OGV and the support plate in TRF is analyzed numerically. The modification in the meridional flow path and the blade load distribution has been made to control the corner separation and to improve the aerodynamic performance. The following conclusions are drawn:

1) When the maximum flow area ratio of the blade area is greater than or equal to 1.053 (the meridional flow path design $\mathrm{A}$ and $\mathrm{B}$ ), the TRF/OGV has a corner separation.

2) When the ratio of the maximum flow area of the blade area is less than or equal to 1.024 , adopting meridional flow path design $\mathrm{C}$ in which the flow area of the blade in the blade area is first expanded and then contracted can create acceleration zone of the trailing edge of OGV, and the threedimensional corner separation of the suction side can be eliminated, the turbine efficiency (include OGV) is 6.5\% higher than the prototype, and the total pressure recovery coefficient of OGV is 0.977 , which is 0.084 higher than the prototype.

3) Due to a more backward design in the maximum deflection position of uniform-loaded airfoil (airfoil design II), the turning is gentler, thus the axial adverse-pressure gradient in the OGV is low, which is beneficial to eliminate corner separation, but the correction of the flow direction is weak. Front-loaded blade type (airfoil design I) has a maximum deflection position close to the maximum thickness position, the blade channel area expands rapidly after the maximum deflection position, and the axial adverse pressure gradient is larger, causing the suction surface corner separation to generate. When adopting the transition-loaded airfoil in which front-loaded in the tip and uniform-loaded in the root (airfoil design III), and the maximum deflection position of the blade is in the middle of the two designs, so that the turning of the suction surface is gentle, and axial length to undertake the pressure gradient increases, which can 
suppress the corner separation and optimally control the direction of the airflow.

4) In the blade design III, the blade load distribution form changes from root to tip, forming the endwall bend in the root, which reduces the lateral pressure gradient of the flow channel, and has a good inhibitory effect on the suction surface corner vortex. The turbine efficiency (include OGV) is $6.4 \%$ higher than that of the prototype. The total pressure recovery coefficient of the $\mathrm{OGV}$ is 0.976 , which is 0.083 higher than the prototype, the outlet flow deviation after OGV is less than $3.5^{\circ}\left(15.864^{\circ}\right.$ for prototype).

\section{REFERENCES}

[1] Zhou Zhengping, et al.Turbine Aerodynamics for Aeroengine: Flow Analysis and Aerodynamics Design[M]. Shanghai Jiao Tong University Press, 2014

[2] Hjärne J, Chernoray V, Larsson J. An Experimental Investigations of Secondary Flows and Loss Development Downstream of a Highly Loaded Low Pressure Turbine Outlet Guide Vane Cascade[R]. ASME Paper GT2006-90561, 2006. [3] Hjärne J, Chernoray V, Larsson J, Löfdahl L. Numerical Validation of Secondary Flows and Loss Development Downstream of a Highly Loaded Low Pressure Turbine Outlet Guide Vane Cascade[R]. ASME Paper GT2007-27712, 2007. [4] Hjärne J, Chernoray V, Larsson J, Löfdahl L. Performance and Off-Design Characteristics for Low Pressure Turbine Outlet Guide Vanes: Measurements and Calculations[R]. ASME Paper GT2006-90550, 2006.

[5] Sonoda T, Schreiber H A, Arima T. Endwall performance of outlet guide vane cascades with different blade loading distributions[R]. ASME Paper GT2008-5111, 2008.

[6]Koch H, Kozulovic D, Hoeger M. Outlet Guide Vane Airfoil for Low Pressure Turbine Configurations[R]. AIAA Paper 2012-2979, 2012

[7] Yan Chen, Research on Some Issues About High Bypass Ratio Turfan Engine Turbine [D]. Beijing University, 2013.

[8] Zhang Yan. Internal Flow and Performance Research on the Intermediate Turbine Duct [D]. Beijing University, 2014

[9] Feng Yahui. Experimental Research on Flow Field and Performance of Turbine Rear Frame [J]. Journal of Engineering Thermophysics [J], 2017, 38(5):941-949.

[10] Feng Yahui. Experimental Research on Flow Field and Performance of Turbine Rear Frame [D]. Beijing University, 2017

[11] Li Jinwei. Aerodynamic Optimization Design and Numerical Simulation of a Single Stage Axial Flow Turbine [D]. Beijing University, 2017

[12] R. Senthil Kumaran, et al. Effect of Axial Velocity Density Ratio on the Performance of a Controlled Diffusion Airfoil Compressor Cascade[J]. International Journal of Turbo \& Jet-Engines,2015,32(4).

[13] Gui Mingmin, et al. Compressor Aerothermodynamics and its Applications in Aircraft Engines [M]. Shanghai Jiao Tong University Press, 2014 